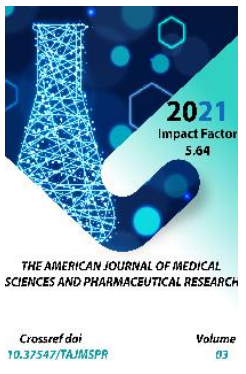

\title{
The Role Of Leptin And Adiponectin In Disorders Of Fatter Tissue In Metabolic Syndrome
}

Nelli Rafikovna Uzbekova

Professor, Candidate Of Medical Sciences, Andijan State Medical Institute, Uzbekistan

\section{ABSTRACT}

In this article, opinions were expressed on the improvement of early-stage diagnostic factors and the optimal pathways of therapeutic treatment, having determined the characteristics of heterogeneous neurohumorals of metabolic syndrome, the clinical manifesto of the disease. The analysis of the study shows that the issues of pathogenesis, clinical features of improving the effectiveness of medicamentous therapy, and treatment-rehabilitation aspects have not been studied perfectly so far. In patients with metabolic syndrome, hypotensive, anti-adipose agents, as well as treatment of statins and improvement of the prophylactic order of prevention of diseases, the issues of which were not studied at the end were discussed.

\section{KEYWORDS}

The hormone, adiponectin, tissue, metabolic syndrome, dietary lipids, plasma concentrations, insulin.

\section{INTRODUCTION}

The main pathogenetic mechanisms of the development of metabolic syndrome are related to the interaction of adipose tissue secretory activity, neurohumoral, adrenergic, inflammatory mechanisms, cardiovascular remodeling, as well as the degree of their participation in the progression of MS. A high coefficient of free leptin and a low level of 
leptin receptors have been shown to cause the mechanism of early development of leptin resistance. The role of high-level ANCA antineutrophil autoantibodies as predictors in the early stages of ED development in patients with metabolic syndrome has been demonstrated in patients with metabolic syndrome, as well as in early remodeling of the cardiovascular system. The effect of high levels was determined.

With the early development of left ventricular hypertrophy, the predictor of cardiac remodeling and an increase in the level of autoantibodies to COM-02 cardiomyocytes, which increase the rate of myocardial dystrophy, have been shown.

Blockade of insulin receptors by autoantibodies has been identified as a mechanism for the development of insulin resistance. The system of treatment of patients with MS diagnosed with arterial hypertension, obesity, dyslipidemia with antihypertensive, anti-adipose and hypolipidemic drugs is pathogenetically based on the desire to improve the complex therapy of MS.

\section{MATERIAL AND METHODS}

For 32 years since $X$ syndrome was described by the American therapist $G$. Reaven, the problem of MS has aroused great interest among scientists and a number of foreign scientists have conducted research on the problem of metabolic syndrome. Although many studies have been conducted today on the problem of MS, a number of issues related to the pathogenesis, diagnosis, and treatment of MS remain unresolved. This is due, on the one hand, to the fact that the mechanisms of formation of the main components of MS are not fully understood, on the other hand, there are objective difficulties of early diagnosis. According to leading experts, the primary link in the mechanism of MS development has not been fully elucidated. Consistent consideration of issues such as neurohumoral factors in the development of MS, nonspecific inflammation, the importance of associated metabolic and structural functional disorders, the extent to which these processes are reversible under the influence of complex therapy is required. The basic criteria for early diagnosis and prognosis of MS have not been improved. The pathogenetic aspects of the sympathetic adrenal system, which lead to the formation of adrenergic imbalances leading to $M S$ in conditions of insulin resistance, leading to chronic activation, are insufficiently dissolved. The interaction of cardiovascular remodeling with the role of IFR-1, neutrophil autoantibodies, and cardiomyocytes has not been established. The issue of drug exposure to key components of MS requires constant study. I.E. Chazova, V.B. According to Mychka, the heterogeneous state of MS poses a great risk for the development of cardiovascular disease. Accordingly, it is recommended to conduct MS diagnosis, complex treatment and prevention in a wide range of medical practice. For the success of a preventive program, the application of modern editing to educational programs and modifying risk factors is of fundamental importance. Analyzes show that the issues of pathogenesis, clinical features and therapeutic-rehabilitation aspects of improving the effectiveness of drug treatment have not yet been fully studied. Improvements in the treatment and prevention of disease with the help of antihypertensive, anti-adipose drugs and statins in patients with metabolic syndrome have not been studied.

\section{RESULT AND DISCUSSION}

The aim of the study was to improve the early diagnostic factors and optimize therapeutic 
approaches by identifying the features of the heterogeneous neurohumoral mechanisms of metabolic syndrome, the clinical manifestation of the disease. Based on patient questionnaires, screening and complex examinations, a step-by-step algorithm for early diagnosis of MS was developed, angiotensin-II receptor blockers (ARBII) (valsartan), I1-imidazoline receptor agonists (moxonidine), adipose drugs (orlistat), 4 generation statins ( an algorithm for improving the therapeutic correction of MS was developed, taking into account the monitoring of therapy with the use of rosuvastatin) and the degree and presence of clinical manifestations of the disease. The reliability of the results of the study is based on the theoretical approach, methods and methodological accuracy of the research, the adequacy of the number of patients, the use of statistical methods, the comparison of international and local experience in determining the specific patterns of clinical metabolic parameters in metabolic syndrome. and based on the fact that the results obtained are confirmed by the competent authorities. Conclusions and recommendations based on the scientific significance of the results of the study are based on the mechanisms of pathogenesis of MS, heterogeneity, neurohumoral, adrenergic imbalances, the role of prooxidant insulinemia controlled by AS parameters, development of endothelial dysregulation, cardiovascular remodeling, YuKTT-ICR-1, IFR-1 is explained by the activation of disease predictors.

The practical significance of the results of the study is that in clinical practice in the treatment of metabolic syndrome plays an important role in the optimization of pathogenetic response in the effective complex treatment of MS components - AG, obesity, DLP with the introduction of $A R B$ II and imidazoline receptor blockers, anti-adipose drugs, 4 generation statins.

Based on the results of research on the neurohumoral mechanism and therapeutic methods of MS pathogenesis: a guideline on "Increased adipose tissue activity in metabolic syndrome and correction of obesity" developed with the help of orlistat to identify ways to correct obesity was approved (Reference of the Ministry of Health No. 8n / 08 of June 15, 2015). Based on the results obtained, the positive effect of orlistat against adipose was shown, which allowed to reduce the level of obesity and its complications. The methodical manual on complex therapy of dyslipidemia in metabolic syndrome "Therapeutic possibilities of correction of dyslipidemia in metabolic syndrome" is approved. Based on the results obtained, it was found that the inclusion of statin 4generation (rosuvastatin) in the treatment schedule had a positive effect, reducing mortality from disability and cardiovascular complications.

Determining the variability of the clinical manifestations of metabolic syndrome, improving the factors of early diagnosis and optimizing the methods of therapeutic analysis given Based on the results obtained, timely diagnosis of MS, reduction of the period of incapacity for work by $20.8 \%$, reduction of the cost of drug treatment by $21.0 \%$, doubling the effectiveness of rehabilitation measures, a positive impact on the quality of life of patients opportunities have been created.

In the concluding part, a comprehensive study of the interaction of adipose tissue secretory activity, neurohumoral mechanisms, systemic inflammatory process, endothelial status, cardiac remodeling revealed heterogeneity of MS pathogenesis. One of the mechanisms of leptin-resistance in the background of hyperleptinemia in metabolic syndrome was an 
increase in the free leptin coefficient and a decrease in OV-R-leptin receptors. An increase in the free leptin coefficient was found to characterize the latent onset of leptin resistance formation $(r=-0.8474 ; r=0.0001)$. Activation of the hormonal mediator joint of the sympathetic-adrenal system in metabolic syndrome has been shown to be associated with increased $K A$, especially noradrenaline and adrenaline biosynthesis. In the body there is a decrease in the activity of the main enzyme of deamination of monoamine oxidases $C A(r=$ $-0.6638 ; r=0.0001)$, which is associated with an increase in the oxidative processes of lipid peroxidation, the qualitative reversible change in the catalytic properties of its activity.

\section{REFERENCES}

1. Uzbekova N.R. Modern concepts of insulin-like growth factors in normal conditions and their role in diabetes mellitus . Volume: 02 Issue: 02 | MarchApril 2021 ISSN: 2660-4159 pp.236-239. www.cajmns.centralasianstudies.org/inde x.php

2. Uzbekova N.R. Neuro humoral mechanisms of the pathogenesis of the metabolic syndrome and ways of their therapeutic correction. Abstract of doctoral (DsC) dissertation on medical sciences. 2017 year.

3. Bagdade J.D., Buchanan W.F., Pollare T., LithellH.Abnormal lipoprotein phospholidip composition in patients with essential hypertension // Atherosclerosis 2009.-117: pp.209-215 .

4. Bruns M.W., Hall J.E. Hyperinsulinemia and hypertonia. NMCD 2003; 3: pp.155-157.

5. Despres J.P. The insulin resistancedyslipidemic syndrome of visceral obesity: effect on patients risk. Obesity Res. 2008; 6: pp.89-179.
6. Esler M., Rumanier., Wiesner G. et al. Sympathetic nervous system and insylin resistance. From obesite to diabetes // Am. J. Hypertens. 2008-14: pp.304-309.

7. Camus J. Goutte, diabete, hyperlipemie: un trisyndrome metaboliqul. Rev Rhumat. 2006; 33:pp.10-14.

8. Cusi K., Defronzo R.A. Metgormin: a revien of its metabolic effects. Diabetes Reviews. 2004; 6: pp.89-131.

9. Reaven G. M. Insulin resistance/compensatory hyperinsulinimia, essential hypertension, and cardiovascular disease. J. ClinEndocrindMetab. 2007;

10. Heseeler J.R., Morel D.W., Leves J., Chisolm G.M., Lipoprotens oxidation and lipoprotein-indused cytotoxicity. // Atherosclerosis. 2006; 3: pp215-222

11. Froze E. Age related changes in postprandial plasma glucose, insulin and FFA concentrations in non-diabetic individuals / E. Froze, M. Chiou, Y. Chen, G.M. Raeven // J Am Geriatr Soc.-2011.- Vol. 35,- pp. 224-228.

12. Furchgott R.F. The obligatoryrole of endotnelial cells in the relazation of arterial smooth muscle by acetylcholine / R.F. Furchgott, J.V. Zawadszki // Nature.2009.- Vol. 288.- pp. 373-376.

13. Anize Delfino von Frankenberg. Relationships between adiponectin levels, the metabolic syndrome, and type 2 diabetes: a literature review.

http://www.scielo.br/scielo.php?script=sci serial\&pid=2359-3997\&lng=en\&nrm issn 2359-4292. 2017 year 